\title{
Mathematical modelling of vertical and longitudinal-angular vibrations in the composition of the rigidity and damping of the hierarchical parts of the tractor taking into account road impact
}

\author{
Farkhod Matmurodov, and Dilafruz Ermatova* \\ Tashkent Institute of Irrigation and Agricultural Mechanization Engineers, Tashkent, Uzbekistan
}

\begin{abstract}
The article contains generalized systems of equations describing the vertical and longitudinal-angular vibrations of the main parts of the tractor with the mathematical model of the hierarchical vertical linear oscillation of the tractor, the irregularities of the roads, taking into account the rigidity and damping. It includes systems: equations describing the vertical vibrations of the skeleton over the propellers of a tractor on an uneven road; equations describing the vertical vibrations of the cab of the rear of the tractor, taking into account the walking of the tractor on uneven roads. Equation systems have also been compiled that describe the vertical oscillations of the uppermost hierarchical link - the seat of the tractor operator, taking into account the walking of the tractor on an uneven road. The use of this original mathematical model will make it possible to find random unwanted oscillations of the new designed machine design. It will help to optimize their geometric and dynamic parameters.
\end{abstract}

\section{Introduction}

The calculation method of the lower rubber damper of the cab allows you to determine the geometric dimensions of the mechanical sub-cab damper.

Using the definition of the vibration of a linear system with one degree of freedom of the under-cab shock absorber of a tractor, we can find the coefficient of efficiency of vibration isolation, which notifies the presence or absence of vibration of the operator's cab.

By numerically solving these equations, we can find the angular and vertical displacement and angular velocity of the cab and the main parts of the tractor. This means that it will be found how much the deflation of the main parts is displaced during vibration.

The article [1] describes theoretical methods for damping low-frequency vibrations on the driver's seat of a wheeled tractor based on the development of mathematical models with various elastic dissipative characteristics of the suspension.

\footnotetext{
*Corresponding author : sardoraka@mail.ru
} 
Methods of incorporating damping relaxation elements into the suspension for shifting resonant vibrations to the frequency range greater than $1 \ldots 8 \mathrm{~Hz}$, as well as methods allowing the practical implementation of the inclusion of

Creating an effective vibration protection system for a wheeled tractor driver is an urgent task [2] aimed at improving the driver's work and ensuring safety.

Seat acceleration and noise levels in the driver's cab of a wheeled vehicle are strictly regulated by international standards. Therefore, global manufacturers of wheeled vehicles are constantly improving vibration protection systems and introducing new absorbing materials in the design of driver's cabins. Thus, studies [2] have determined the frequency ranges of natural vibrations of various human organs and established the distribution of vibration frequencies over the structural elements of a wheeled tractor.

F. Deine and M. Michke pay special attention to low-frequency vibrations $(1 \ldots 8 \mathrm{~Hz})$, which have the most harmful effect on the driver and cause him occupational diseases.

Studies have established that the natural frequency of human vibrations on the seat of modern wheeled tractors is $13 \mathrm{~Hz}$, and the permissible values of the root-mean-square accelerations and amplitudes of vertical movements of the seat should not exceed $8.5 \mathrm{~m} / \mathrm{s} 2$ and $0.02 \mathrm{~m}$, respectively, which is stipulated by GOST 12.1. 012-90 and Council Directive No. $78 / 764$.

\section{Research Methods}

The equations describing vertical and longitudinal-angular oscillations are written according to the Lagrange equation of the second kind.

In the mathematical modelling of the hierarchical vertical linear oscillation of the tractor, taking into account the unevenness of the roads, the stiffness and damping coefficient is considered.

\section{Results and discussion}

The power load and the speed of the tractors are constantly increasing, which leads to an increase in the dynamic load of the main parts and an increase in the level of oscillations generated by them. Vibration loads have a negative effect on the components and parts of the tractor, on the environment, and on the cab with the operator.

The main parts of the tractor are described by the flat, created at the first stage, and at the subsequent stage, the spatial model of the suspension systems [1]. 


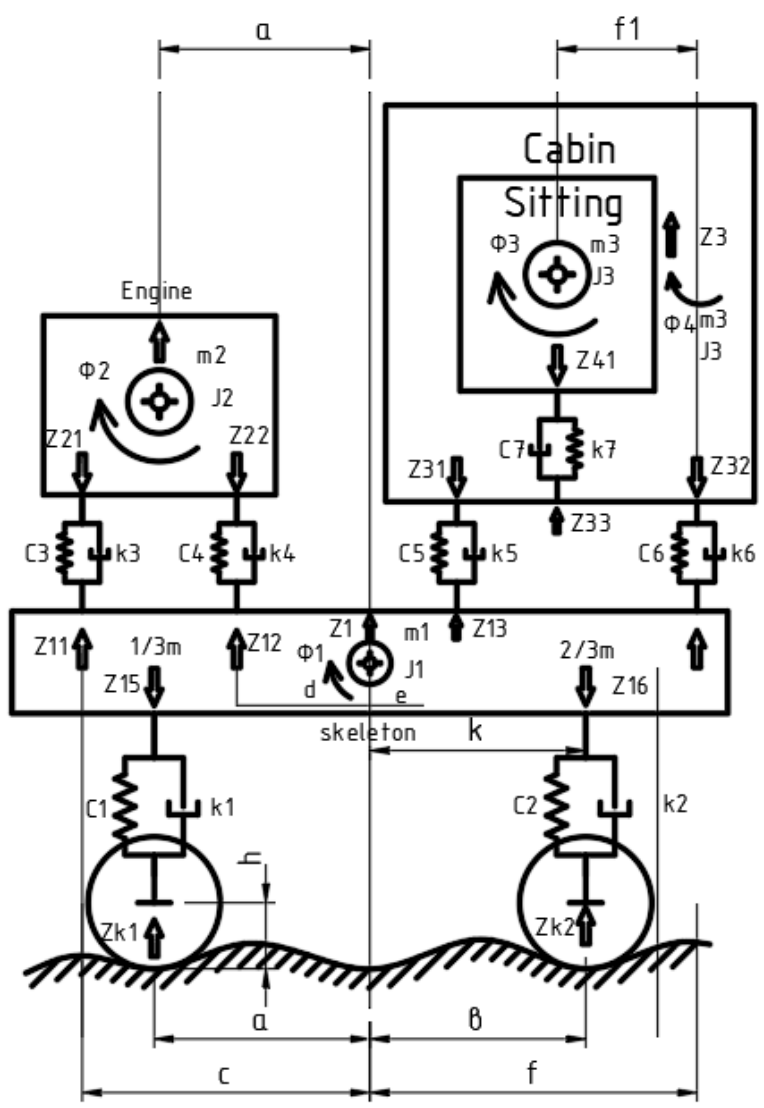

Fig. 1. Dynamic equivalent design of the main parts of the tractor

a) equations describing vertical oscillations

$$
\begin{gathered}
\left\{\begin{array}{c}
m_{1} \ddot{z}_{1}-\left[2 c_{1}\left(z_{15}-h \cos \left(\omega_{1} t\right)\right)+2 k_{1}\left(\dot{z}_{15}-d\left(h \cos \left(\omega_{1} t\right)\right) / d t\right)\right]+ \\
{\left[2 c_{2}\left(z_{16}-z_{k 2}\right)+2 k_{2}\left(\dot{z}_{16}-\dot{z}_{k 2}\right)\right]-} \\
{\left[2 c_{3}\left(z_{21}-z_{11}\right)+2 k_{3}\left(\dot{z}_{21}-\dot{z}_{11}\right)\right]-\left[2 c_{4}\left(z_{22}-z_{12}\right)+2 k_{4}\left(\dot{z}_{22}-\dot{z}_{12}\right)\right]+} \\
{\left[2 c_{5}\left(z_{31}-z_{13}\right)+2 k_{5}\left(\dot{z}_{31}-\dot{z}_{13}\right)\right]-\left[2 c_{6}\left(z_{32}-z_{14}\right)+2 k_{6}\left(\dot{z}_{32}-\dot{z}_{14}\right)\right]=m_{1} g,} \\
m_{2} \ddot{z}_{2}+\left[2 c_{3}\left(z_{21}-z_{11}\right)+2 k_{3}\left(\dot{z}_{21}-\dot{z}_{11}\right)\right]+\left[2 c_{4}\left(z_{22}-z_{12}\right)+2 k_{4}\left(\dot{z}_{22}-\dot{z}_{12}\right)\right]=m_{2} g, \\
m_{3} \ddot{z}_{3}+\left[2 c_{5}\left(z_{31}-z_{13}\right)+2 k_{5}\left(\dot{z}_{31}-\dot{z}_{13}\right)\right]+\left[2 c_{6}\left(z_{32}-z_{14}\right)+2 k_{6}\left(\dot{z}_{32}-\dot{z}_{14}\right)\right]- \\
{\left[2 c_{7}\left(z_{41}-z_{32}\right)+2 k_{7}\left(\dot{z}_{41}-\dot{z}_{32}\right)\right]=m_{3} g}
\end{array}\right. \\
m_{4} \ddot{z}_{4}+\left[2 c_{7}\left(z_{41}-z_{32}\right)+2 k_{7}\left(\dot{z}_{41}-\dot{z}_{32}\right)\right]=m_{4} g \\
\left\{\begin{array}{c}
m_{1} \ddot{z}_{1}-\left[2 c_{1}\left(z_{15}-z_{k 1}\right)+2 k_{1}\left(\dot{z}_{15}-\dot{z}_{k 1}\right)\right]+\left[2 c_{2}\left(z_{16}-z_{k 2}\right)+2 k_{2}\left(\dot{z}_{16}-\dot{z}_{k 2}\right)\right]- \\
{\left[2 c_{3}\left(z_{21}-z_{11}\right)+2 k_{3}\left(\dot{z}_{21}-\dot{z}_{11}\right)\right]-\left[2 c_{4}\left(z_{22}-z_{12}\right)+2 k_{4}\left(\dot{z}_{22}-\dot{z}_{12}\right)\right]+} \\
{\left[2 c_{5}\left(z_{31}-z_{13}\right)+2 k_{5}\left(\dot{z}_{31}-\dot{z}_{13}\right)\right]-\left[2 c_{6}\left(z_{32}-z_{14}\right)+2 k_{6}\left(\dot{z}_{32}-\dot{z}_{14}\right)\right]=m_{1} g,} \\
m_{2} \ddot{z}_{2}+\left[2 c_{3}\left(z_{21}-z_{11}\right)+2 k_{3}\left(\dot{z}_{21}-\dot{z}_{11}\right)\right]+\left[2 c_{4}\left(z_{22}-z_{12}\right)+2 k_{4}\left(\dot{z}_{22}-\dot{z}_{12}\right)\right]=m_{2} g, \\
m_{3} \ddot{z}_{3}+\left[2 c_{5}\left(z_{31}-z_{13}\right)+2 k_{5}\left(\dot{z}_{31}-\dot{z}_{13}\right)\right]+\left[2 c_{6}\left(z_{32}-z_{14}\right)+2 k_{6}\left(\dot{z}_{32}-\dot{z}_{14}\right)\right]- \\
{\left[2 c_{7}\left(z_{41}-z_{32}\right)+2 k_{7}\left(\dot{z}_{41}-\dot{z}_{32}\right)\right]=m_{3} g} \\
m_{4} \ddot{z}_{4}+\left[2 c_{7}\left(z_{41}-z_{32}\right)+2 k_{7}\left(\dot{z}_{41}-\dot{z}_{32}\right)\right]=m_{4} g
\end{array}\right.
\end{gathered}
$$


b) equations describing longitudinal-angular oscillations

$$
\begin{aligned}
& \left\{\begin{array}{c}
J_{1} \ddot{\varphi}_{1}+\left[2 c_{1}\left(z_{15}-z_{k 1}\right)+2 k_{1}\left(\dot{z}_{15}-\dot{z}_{k 1}\right)\right] a-\left[2 c_{2}\left(z_{16}-z_{k 2}\right)+2 k_{2}\left(\dot{z}_{16}-\dot{z}_{k 2}\right)\right] b- \\
{\left[2 c_{3}\left(z_{21}-z_{11}\right)+2 k_{3}\left(\dot{z}_{21}-\dot{z}_{11}\right)\right] c-\left[2 c_{4}\left(z_{22}-z_{12}\right)+2 k_{4}\left(\dot{z}_{22}-\dot{z}_{12}\right)\right] d+} \\
{\left[2 c_{5}\left(z_{31}-z_{13}\right)+2 k_{5}\left(\dot{z}_{31}-\dot{z}_{13}\right)\right] e-\left[2 c_{6}\left(z_{32}-z_{14}\right)+2 k_{6}\left(\dot{z}_{32}-\dot{z}_{14}\right)\right] f=0,} \\
J_{2} \ddot{\varphi}_{2}+\left[2 c_{3}\left(z_{21}-z_{11}\right)+2 k_{3}\left(\dot{z}_{21}-\dot{z}_{11}\right)\right](c-n)+\left[2 c_{4}\left(z_{22}-z_{12}\right)+2 k_{4}\left(\dot{z}_{22}-\dot{z}_{12}\right)\right](n-d)=0, \varphi_{1} \\
J_{3} \ddot{\varphi}_{3}+\left[2 c_{5}\left(z_{31}-z_{13}\right)+2 k_{5}\left(\dot{z}_{31}-\dot{z}_{13}\right)\right](h-e)+\left[2 c_{6}\left(z_{32}-z_{14}\right)+2 k_{6}\left(\dot{z}_{32}-\dot{z}_{14}\right)\right](f-h) \\
=0, \\
J_{4} \ddot{\varphi}_{4}+\left[2 c_{7}\left(z_{41}-z_{33}\right)+2 k_{7}\left(\dot{z}_{41}-\dot{z}_{33}\right)\right]\left(f-f_{1}\right)=0,
\end{array}\right. \\
& \left\{\begin{array} { l } 
{ z _ { 1 1 } = z _ { 1 } - \varphi _ { 1 } c , } \\
{ z _ { 1 2 } = z _ { 1 } - \varphi _ { 1 } d , } \\
{ z _ { 1 3 } = z _ { 1 } + \varphi _ { 1 } e , } \\
{ z _ { 1 4 } = z _ { 1 } + \varphi _ { 1 } f , } \\
{ z _ { 1 5 } = z _ { 1 } - \varphi _ { 1 } a , } \\
{ z _ { 1 7 } = z _ { 1 } - \varphi _ { 1 } b , }
\end{array} \quad \left\{\begin{array} { l } 
{ z _ { 2 1 } = z _ { 2 } + \varphi _ { 2 } ( c - n ) , } \\
{ z _ { 2 2 } = z _ { 2 } - \varphi _ { 2 } ( n - d ) , } \\
{ z _ { 3 1 } = z _ { 3 } + \varphi _ { 3 } ( h - e ) , } \\
{ z _ { 3 2 } = z _ { 3 } - \varphi _ { 3 } ( f - h ) , } \\
{ z _ { 3 3 } = z _ { 3 } + \varphi _ { 3 } ( h - k ) , } \\
{ d z _ { 1 1 } = d z _ { 1 } - d \varphi _ { 1 } c , } \\
{ d z _ { 1 2 } = d z _ { 1 } - d \varphi _ { 1 } d , }
\end{array} \quad \left\{\begin{array}{r}
d z_{13}=d z_{1}+d \varphi_{1} e, \\
d z_{14}=d z_{1}+d \varphi_{1} f \\
d z_{15}=d z_{1}+d \varphi_{1} a, \\
d z_{16}=d z_{1}-d \varphi_{1} b \\
d z_{21}=d z_{2}+d \varphi_{2}(c-n), \\
d z_{22}=d z_{2}-d \varphi_{2}(n-d), \\
d z_{31}=d z_{3}+d \varphi_{3}(h-e), \\
d z_{32}=d z_{3}-d \varphi_{3}(f-h), \\
d z_{33}=d z_{3}+d \varphi_{3}(h-k)
\end{array}\right.\right.\right.
\end{aligned}
$$

Here mi is the $\mathrm{i}$-th sprung mass; Ji-moment of inertia of the $\mathrm{i}$-th sprung mass; sprung mass, $z_{i}, z_{i}, z_{i}$ are respectively vertical displacement, speed, acceleration, i-th sprung mass; $d z_{i}$ are corresponding speeds of vertical displacements; $\varphi_{i}, \varphi_{i}, \varphi_{i}{ }_{i}$ are angular displacement, speed, acceleration, i-th sprung mass; $c_{i}$ i-th bond stiffness; $k_{i}$ i-th damping factor; a, b, c, d, $\mathrm{e}, \mathrm{f}, \mathrm{h}, \mathrm{k}, \mathrm{n}$ are geometric parameters of elements; $\mathrm{g}$ is the acceleration of gravity.

Based on a flat model, when using an algorithmic program, it is possible to obtain the oscillatory systems of the frame, cab, and seat and graphs of displacements, speeds, and accelerations of these tractor parts.

Mathematical modeling of the hierarchical vertical linear oscillation of the tractor, taking into account the unevenness of the roads, considering the coefficient of stiffness and damping.

Oscillations in machines are often undesirable, as they reduce the reliability of machines, cause noise and have a harmful effect on the human body. The dynamic equivalent total design of the main parts of the tractor is shown in Fig.

At the same time, for the convenience of analytical calculation and creation of the possibility of implementing ideas, we take the following assumptions:

- takes only the stiffness coefficient in the calculation;

- the damping factor is neglected; if we take into account the mathematical model, it becomes too complicated;

- various forced and natural vibrations are considered absent, etc. 


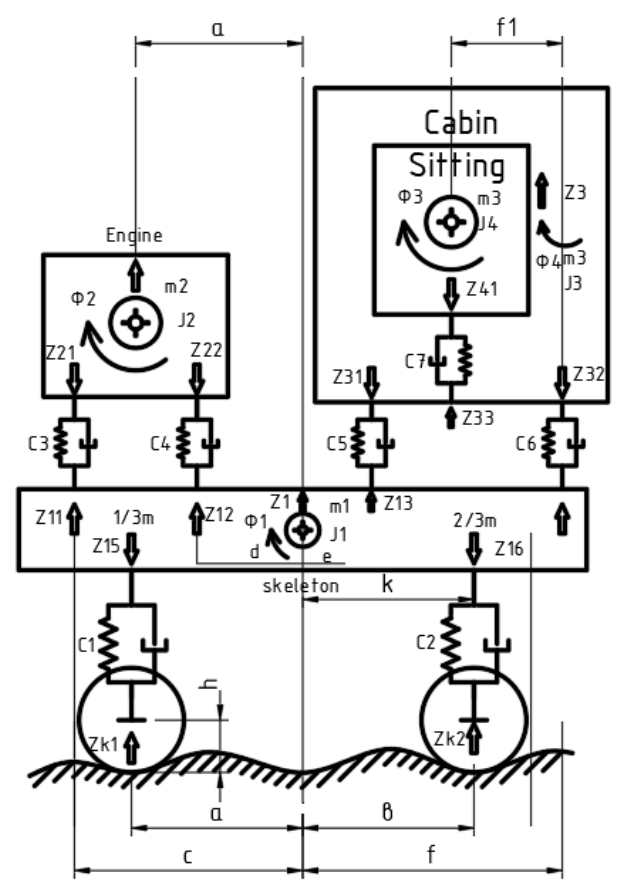

Fig. 2. Dynamic equivalent total calculated main parts of the tractor

a) equations describing the vertical vibrations of the frame over the tractor propellers on an uneven road. Let us consider the effect of oscillation on a mechanical system using a model depicting the movement of a mass of $1 / 3 \mathrm{~m}$ and $2 / 3 \mathrm{~m}$; we assume that a third of the mass falls on the front of the tractor and two of a third of the mass that is elastically suspended on the wheel falls on the rear of the tractor, which rolls along a rigid path with cosine-shaped irregularities (Figure 3). It is obvious that the system will perform forced oscillations.

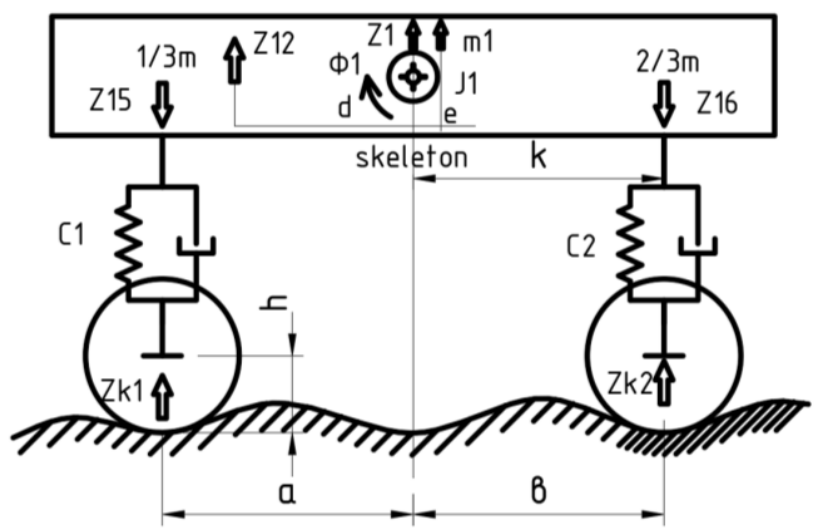

Fig. 3. Carcass over tractor propellers on uneven road

In this system, the forces of inertia of mass m, i.e., mz", are balanced by the forces arising from the deformation of the tire (z-zk), i.e. force $\mathrm{c}(\mathrm{z}-\mathrm{zk})$. 
Consequently,

Define the linear vibration of the machine

$$
\begin{gathered}
\frac{1}{3} m \ddot{x}=\sum_{i=1}^{2}\left(P_{k i}-P_{f i}\right) \cos \beta+\sum_{i=3}^{4}\left(P_{k i}-P_{f i}\right) \cos (\beta+\alpha)+ \\
\sum_{i=1}^{2}\left(P_{b i}-P_{b f i}\right) \sin \beta+\sum_{i=3}^{4}\left(P_{b i}-P_{b f i}\right) \sin (\beta+\alpha)-2 c_{t}(x-\beta h)-2 k_{t}(\dot{x}-\dot{\beta} h) \\
-c_{1}\left(z_{15}-h \cos \left(\omega_{1} t\right)\right)+k_{1}\left(\dot{z}_{15}-\frac{d\left(h \cos \left(\omega_{1} t\right)\right)}{d t}\right)+\left(P_{k r i} \cos (\beta-\alpha)\right. \\
\left.-P_{c i} \cos (\beta-\alpha)\right)+\frac{m_{M} j_{M}+m_{i s} j_{i s}}{2}
\end{gathered}
$$

In the drawing of the spatial model, add the bump height of the roads

$$
\left\{\begin{array}{l}
\frac{1}{3 m} \ddot{z}_{15}+c_{15}\left(z_{15}-z_{k 1}\right)+k_{15}\left(\dot{z}_{15}-\dot{z}_{k 1}\right)=0 \\
\frac{2}{3 m} \ddot{z}_{16}+c_{16}\left(z_{16}-z_{k 2}\right)+k_{16}\left(\dot{z}_{16}-\dot{z}_{k 2}\right)=0
\end{array}\right.
$$

If a $z_{k 1}=h \cos \left(\omega_{1} t\right), z_{k 2}=h \cos \left(\omega_{2} t\right)$ то

$$
\left\{\begin{array}{l}
\frac{1}{3} m \ddot{z}_{15}+c_{15}\left(z_{15}-h \cos \left(\omega_{1} t\right)\right)-k_{15}\left(\dot{z}_{15}-d\left(h \cos \left(\omega_{1} t\right)\right) / d t\right)=0 \\
\frac{2}{3} \ddot{m} z_{16}+c_{16}\left(z_{16}-h \cos \left(\omega_{2} t\right)\right)-k_{16}\left(\dot{z}_{16}-d\left(h \cos \left(\omega_{2} t\right)\right) / d t\right)=0
\end{array}\right.
$$

Dividing all terms of this equation by $\mathrm{m}$, we get

$$
\left\{\begin{array}{l}
\ddot{z}_{15}+v_{15}^{2}\left(z_{15}-h \cos \left(\omega_{1} t\right)\right)=\theta_{15}^{2}\left(\dot{z}_{15}+\omega_{1} h \sin \left(\omega_{1} t\right)\right)=0 \\
\ddot{z}_{16}+v_{16}^{2}\left(z_{16}-h \cos \left(\omega_{2} t\right)\right)=\theta_{16}^{2}\left(\dot{z}_{16}+\omega_{2} h \sin \left(\omega_{2} t\right)\right)=0
\end{array}\right.
$$

Where $v_{15}^{2}=c_{15} /(1 / 3 m), v_{16}^{2}=c_{16} /(1 / 3 m), \theta_{15}^{2}=k_{15} /(2 / 3 m), \theta_{16}^{2}=k_{16} /(2 / 3 m)$ is system circular frequency.

The general solution of these equations with the right-hand side (inhomogeneous) can be represented as the sum of the solution of the homogeneous equations $\mathrm{z}_{151}, \mathrm{z}_{161}$, and the particular solution of the inhomogeneous equations $z_{152}, z_{162}$, T.e. $z_{15}=z_{151}+z_{152}, z_{16}=$ $\mathrm{z}_{161}+\mathrm{z}_{162}$.

Let us first find a particular solution to the equation. Imagine that

$$
\left\{\begin{array}{l}
z_{152}=A_{152} \cos \left(\omega_{152} t\right) \\
z_{162}=A_{162} \cos \left(\omega_{162} t\right)
\end{array}\right.
$$

and substitute it into equation (2). As a result, we get

$$
\begin{gathered}
\left\{\begin{array}{c}
\ddot{z}_{15}+v_{15}^{2}\left(z_{15}-h \cos \left(\omega_{1} t\right)\right)=\theta_{15}^{2}\left(\dot{z}_{15}+\omega_{1} h \sin \left(\omega_{1} t\right)\right)=0 \\
\ddot{z}_{16}+v_{16}^{2}\left(z_{16}-h \cos \left(\omega_{2} t\right)\right)=\theta_{16}^{2}\left(\dot{z}_{16}+\omega_{2} h \sin \left(\omega_{2} t\right)\right)=0
\end{array}\right. \\
\left\{\begin{array}{c}
-A_{152} \omega_{152}^{2} \sin \left(\omega_{152} t\right)+v_{15}^{2} A_{152} \cos \left(\omega_{152} t\right)-v_{15}^{2} h \cos \left(\omega_{152} t\right)= \\
-\theta_{15}^{2} \omega_{152} A_{152} \sin \left(\omega_{152} t\right)+\theta_{15}^{2} \omega_{152} h \sin \left(\omega_{152} t\right) \\
-A_{162} \omega_{162}^{2} \sin \left(\omega_{162} t\right)+v_{16}^{2} A_{162} \cos \left(\omega_{162} t\right)-v_{16}^{2} h \cos \left(\omega_{162} t\right)= \\
-\theta_{16}^{2} \omega_{162} A_{162} \sin \left(\omega_{162} t\right)+\theta_{16}^{2} \omega_{162} h \sin \left(\omega_{162} t\right) .
\end{array}\right.
\end{gathered}
$$


From where

$$
\begin{gathered}
\left\{\begin{array}{l}
A_{152}=\left(\theta_{15}^{2} \omega_{152} h-v_{15}^{2} h \operatorname{ctg}\left(\omega_{152} t\right)\right) /\left(\omega_{152}^{2}-v_{15}^{2} \operatorname{ctg}\left(\omega_{152} t\right)+\theta_{15}^{2} \omega_{152}\right) \\
A_{162}=\left(\theta_{16}^{2} \omega_{162} h-v_{16}^{2} h \operatorname{ctg}\left(\omega_{162} t\right)\right) /\left(\omega_{162}^{2}-v_{16}^{2} \operatorname{ctg}\left(\omega_{162} t\right)+\theta_{16}^{2} \omega_{162}\right)
\end{array}\right. \\
\left\{\begin{array}{l}
z_{152}=\left[\left(\theta_{15}^{2} \omega_{152} h-v_{15}^{2} h \operatorname{ctg}\left(\omega_{152} t\right)\right) /\left(\omega_{152}^{2}-v_{15}^{2} \operatorname{ctg}\left(\omega_{152} t\right)+\theta_{15}^{2} \omega_{152}\right)\right] \cos \left(\omega_{152} t\right) \\
z_{162}=\left[\left(\theta_{16}^{2} \omega_{162} h-v_{16}^{2} h \operatorname{ctg}\left(\omega_{162} t\right)\right) /\left(\omega_{162}^{2}-v_{16}^{2} \operatorname{ctg}\left(\omega_{162} t\right)+\theta_{16}^{2} \omega_{162}\right)\right] \cos \left(\omega_{162} t\right)
\end{array}\right.
\end{gathered}
$$

As is known, the solution to a homogeneous equation can be represented as:

$$
\left\{\begin{array}{l}
z_{151}=-\frac{1}{3} A_{151}\left[\cos \left(v_{151} t\right)+\cos \left(\theta_{151} t\right)\right] \\
z_{161}=-\frac{2}{3} A_{161}\left[\cos \left(v_{161} t\right)+\cos \left(\theta_{161} t\right)\right]
\end{array}\right.
$$

Then the general solution of equation (2) is represented as

$$
\left\{\begin{array}{c}
z_{15}=\left[\left(\theta_{15}^{2} \omega_{152} h-v_{15}^{2} h \operatorname{ctg}\left(\omega_{152} t\right)\right) /\left(\omega_{152}^{2}-v_{15}^{2} \operatorname{ctg}\left(\omega_{152} t\right)+\theta_{15}^{2} \omega_{152}\right)\right] \cos \left(\omega_{152} t\right)- \\
-\frac{1}{3} A_{151}\left[\cos \left(v_{151} t\right)+\cos \left(\theta_{151} t\right)\right] \\
z_{16}=\left[\left(\theta_{16}^{2} \omega_{162} h-v_{16}^{2} h \operatorname{ctg}\left(\omega_{162} t\right)\right) /\left(\omega_{162}^{2}-v_{16}^{2} \operatorname{ctg}\left(\omega_{162} t\right)+\theta_{16}^{2} \omega_{162}\right)\right] \cos \left(\omega_{162} t\right)- \\
-\frac{2}{3} A_{161}\left[\cos \left(v_{161} t\right)-\cos \left(\theta_{161} t\right)\right]
\end{array}\right.
$$

The origin of time $(t=0)$ in this system can be taken for such a moment when $z=0$. In this case, substituting $t=0$ and $z=0$ into (4), we obtain

$$
\left\{\begin{array}{l}
\frac{1}{3} A_{151}=\left[\theta_{15}^{2} \omega_{152} h /\left(\dot{\omega}_{152}+\theta_{15}^{2} \omega_{152}+1\right)\right] \\
\frac{2}{3} A_{161}=\left[\theta_{16}^{2} \omega_{162} h /\left(\omega_{162}^{2}+\theta_{16}^{2} \omega_{162}+1\right)\right]
\end{array}\right.
$$

Substituting $\mathrm{A}_{l}$ into (4), we obtain

$$
\left\{\begin{array}{l}
z_{151}=-\left[\theta_{15}^{2} \omega_{152} h /\left(\omega_{152}^{2}+\theta_{15}^{2} \omega_{152}+1\right)\right]\left[\cos \left(v_{151} t\right)+\cos \left(\theta_{151} t\right)\right] \\
z_{161}=-\left[\theta_{16}^{2} \omega_{162} h /\left(\omega_{162}^{2}+\theta_{16}^{2} \omega_{162}+1\right)\right]\left[\cos \left(v_{161} t\right)+\cos \left(\theta_{161} t\right)\right]
\end{array}\right.
$$

The value $\omega_{152} /\left(\omega_{152}^{2}+\theta_{15}^{2} \omega_{152}+1\right)=x_{15}, \omega_{162} /\left(\omega_{162}^{2}+\theta_{16}^{2} \omega_{162}+1\right)=x_{16}$ is called the coefficient of growth of oscillations. Taking this notation, equation (5) will be written

$$
\left\{\begin{array}{l}
z_{15}=-x_{15} \theta_{15}^{2} h\left[\cos \left(v_{151} t\right)+\cos \left(\theta_{151} t\right)\right] \\
z_{16}=-x_{16} \theta_{16}^{2} h\left[\cos \left(v_{161} t\right)+\cos \left(\theta_{161} t\right)\right]
\end{array} .\right.
$$

This will be the general solution to our equation under the initial conditions adopted above.

As $\omega$ approaches $v$, the period $T_{1}, T_{2}$ increases. When the quantities $\omega$ and $v . \theta$ coincide exactly, the phenomenon of resonance occurs.

b) equations describing the vertical vibrations of the cab located at the rear of the tractor, taking into account the walking of the tractor on an uneven road. 
The cab is located at the rear of the tractor. To simplify the analytical calculation, we assume that the vertical vibrations of the front and engine vibrations do not affect the vibrations of the cab and the tractor operator. We believe that the rocking of the rear of the tractor affects the vibrations in the cab and the operator's workplace.

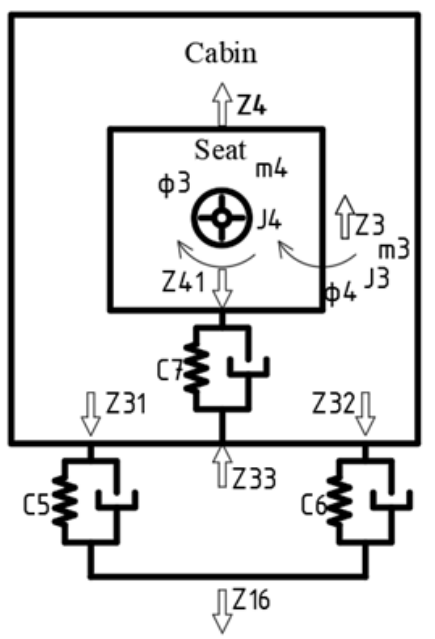

Fig. 4. Vibrations of the cab and operator's workplace

In this system, the forces of inertia are masses $m 3, m 4$, i.e., $m \ddot{z}$, are balanced by the forces arising from the deformation of the tire $\left(z-z_{k}\right)$, i.e., force $\mathrm{c}\left(z-z_{k}\right), k\left(\dot{z}-\dot{z}_{k}\right)$.

Consequently,

for the cab, here the vibration of the rear wheel is mainly influenced by this only takes into account the movements $z_{16}$

$$
\begin{gathered}
\left\{\begin{array}{c}
\frac{1}{2} m_{3} \ddot{z}_{31}+c_{5}\left(z_{31}-z_{16}\right)+k_{5}\left(\dot{z}_{31}-\dot{z}_{16}\right)=0 \\
\frac{2}{2} m_{3} \ddot{z}_{32}+c_{6}\left(z_{32}-z_{16}\right)+k_{6}\left(\dot{z}_{32}-\dot{z}_{16}\right)=0
\end{array}\right. \\
\text { for seat } z_{3}=\left(z_{31}+z_{32}\right)
\end{gathered}
$$

If we take $z_{16}$ from equation (6), then

for cabin

$$
\begin{gathered}
\left\{\begin{array}{c}
\frac{1}{2} m_{3} \ddot{z}_{31}+c_{5} z_{31}+c_{5} x_{16} \theta_{16}^{2} h\left[\cos \left(v_{161} t\right)+\cos \left(\theta_{161} t\right)\right]+k_{5} \dot{z}_{31}+ \\
k_{5} d\left\{x_{16} \theta_{16}^{2} h\left[\cos \left(v_{161} t\right)+\cos \left(\theta_{161} t\right)\right]\right\} / d t=0 \\
\frac{2}{2} m_{3} \ddot{z}_{32}+c_{6} z_{32}+c_{6} x_{16} \theta_{16}^{2} h\left[\cos \left(v_{161} t\right)+\cos \left(\theta_{161} t\right)\right]+k_{6} \dot{z}_{32}+ \\
k_{6} d\left\{x_{16} \theta_{16}^{2} h\left[\cos \left(v_{161} t\right)+\cos \left(\theta_{161} t\right)\right]\right\} / d t=0
\end{array}\right. \\
\left\{\begin{array}{c}
\frac{1}{2} m_{3} \ddot{z}_{31}+c_{5} z_{31}+c_{5} x_{16} \theta_{16}^{2} h\left[\cos \left(v_{161} t\right)+\cos \left(\theta_{161} t\right)\right]+k_{5} \dot{z}_{31}- \\
k_{5} x_{16} \theta_{16}^{2} h\left[v_{161} \sin \left(v_{161} t\right)+\theta_{161} \sin \left(\theta_{161} t\right)\right]=0 \\
\frac{2}{2} m_{3} \ddot{z}_{32}+c_{6} z_{32}+c_{6} x_{16} \theta_{16}^{2} h\left[\cos \left(v_{161} t\right)+\cos \left(\theta_{161} t\right)\right]+k_{6} \dot{z}_{32}- \\
k_{6} x_{16} \theta_{16}^{2} h\left[v_{161} \sin \left(v_{161} t\right)+\theta_{161} \sin \left(\theta_{161} t\right)\right]=0
\end{array}\right.
\end{gathered}
$$


Dividing all terms of this equation by $1 / 2 \mathrm{~m}_{3}$, we get

$$
\left\{\begin{array}{c}
\ddot{z}_{31}+v_{5}^{2} z_{31}+v_{5}^{2} x_{16} \theta_{16}^{2} h\left[\cos \left(v_{161} t\right)+\cos \left(\theta_{161} t\right)\right]+\theta_{5}^{2} \dot{z}_{31}- \\
\theta_{5}^{2} x_{16} \theta_{16}^{2} h\left[v_{161} \sin \left(v_{161} t\right)+\theta_{161} \sin \left(\theta_{161} t\right)\right]=0 \\
\ddot{z}_{32}+v_{6}^{2} z_{32}+v_{6}^{2} x_{16} \theta_{16}^{2} h\left[\cos \left(v_{161} t\right)+\cos \left(\theta_{161} t\right)\right]+\theta_{6}^{2} \dot{z}_{32}- \\
\theta_{6}^{2} x_{16} \theta_{16}^{2} h\left[v_{161} \sin \left(v_{161} t\right)+\theta_{161} \sin \left(\theta_{161} t\right)\right]=0
\end{array}\right.
$$

where $v_{5}^{2}=c_{5} /\left(1 / 2 m_{3}\right), v_{6}^{2}=c_{6} /\left(1 / 2 m_{3}\right), \theta_{5}^{2}=k_{5} /\left(1 / 2 m_{3}\right), \theta_{6}^{2}=k_{6} /\left(1 / 2 m_{3}\right)$ are circular frequencies of free vibrations of the cabin.

The general solution of these equations with the right-hand side (inhomogeneous) can be represented as the sum of the solution of the homogeneous equations $z_{311}, z_{321}$, and the particular solution of the inhomogeneous equations $\mathrm{z}_{312}, \mathrm{z}_{322}$, т.e. $\mathrm{z}_{31}=\mathrm{z}_{311}+\mathrm{z}_{312}, \mathrm{z}_{32}=$ $\mathrm{z}_{321}+\mathrm{z}_{322}$.

Let us first find a particular solution to the equation. Imagine that

$$
\left\{\begin{array}{l}
z_{312}=A_{312}\left[\cos \left(\omega_{312} t\right)+\cos \left(\theta_{312} t\right)\right] \\
z_{322}=A_{322}\left[\cos \left(\omega_{322} t\right)+\cos \left(\theta_{322} t\right)\right]
\end{array}\right.
$$

and substitute it into equation (13). As a result, we get

$$
\begin{aligned}
& \left\{\begin{array}{l}
-\frac{1}{2} A_{312} \omega_{312}^{2} \cos \left(\omega_{312} t\right)+v_{5}^{2} A_{312} \cos \left(\omega_{312} t\right)=v_{5}^{2} \frac{3 \omega_{162} h}{4 \varepsilon_{2}} t \sin \varepsilon_{2} t \\
-\frac{1}{2} A_{322} \omega_{322}^{2} \cos \left(\omega_{322} t\right)+v_{6}^{2} A_{322} \cos \left(\omega_{322} t\right)=v_{6}^{2} \frac{3 \omega_{162} h}{4 \varepsilon_{2}} t \sin \varepsilon_{2} t
\end{array}\right. \\
& \left\{\begin{array}{c}
-A_{312}\left[\omega_{312}^{2} \cos \left(\omega_{312} t\right)+\theta_{312}^{2} \cos \left(\theta_{312} t\right)\right]+v_{5}^{2} A_{312}\left[\cos \left(\omega_{312} t\right)+\cos \left(\theta_{312} t\right)\right]+ \\
v_{5}^{2} x_{16} \theta_{16}^{2} h\left[\cos \left(v_{161} t\right)+\cos \left(\theta_{161} t\right)\right]-\theta_{5}^{2} A_{312}\left[\omega_{312} \sin \left(\omega_{312} t\right)+\theta_{312} \sin \left(\theta_{312} t\right)\right]- \\
\theta_{5}^{2} x_{16} \theta_{16}^{2} h\left[v_{161} \sin \left(v_{161} t\right)+\theta_{161} \sin \left(\theta_{161} t\right)\right]=0 \\
-A_{322}\left[\omega_{322}^{2} \cos \left(\omega_{322} t\right)+\theta_{322}^{2} \cos \left(\theta_{322} t\right)\right]+v_{6}^{2} A_{322}\left[\cos \left(\omega_{322} t\right)+\cos \left(\theta_{322} t\right)\right]+ \\
v_{6}^{2} x_{16} \theta_{16}^{2} h\left[\cos \left(v_{161} t\right)+\cos \left(\theta_{161} t\right)\right]-\theta_{6}^{2} A_{322}\left[\omega_{322} \sin \left(\omega_{322} t\right)+\theta_{322} \sin \left(\theta_{322} t\right)\right]- \\
\theta_{6}^{2} x_{16} \theta_{16}^{2} h\left[v_{161} \sin \left(v_{161} t\right)+\theta_{161} \sin \left(\theta_{161} t\right)\right]=0
\end{array}\right. \\
& \left\{\begin{array}{c}
A_{312}=\left\{-v_{5}^{2} x_{16} \theta_{16}^{2} h\left[\cos \left(v_{161} t\right)+\cos \left(\theta_{161} t\right)\right]+\theta_{5}^{2} x_{16} \theta_{16}^{2} h\left[v_{161} \sin \left(v_{161} t\right)+\right.\right. \\
\left.\left.\theta_{161} \sin \left(\theta_{161} t\right)\right]\right\} /\left\{\left[\omega_{312}^{2} \cos \left(\omega_{312} t\right)+\theta_{312}^{2} \cos \left(\theta_{312} t\right)\right]-v_{5}^{2}\left[\cos \left(\omega_{312} t\right)+\right.\right. \\
\left.\cos \left(\theta_{312} t\right)\right]+\theta_{5}^{2}\left[\omega_{312} \sin \left(\omega_{312} t\right)+\theta_{312} \sin \left(\theta_{312} t\right)\right] \\
A_{322}=\left\{-v_{6}^{2} x_{16} \theta_{16}^{2} h\left[\cos \left(v_{161} t\right)+\cos \left(\theta_{161} t\right)\right]+\theta_{6}^{2} x_{16} \theta_{16}^{2} h\left[v_{161} \sin \left(v_{161} t\right)+\right.\right. \\
\left.\left.\theta_{161} \sin \left(\theta_{161} t\right)\right]\right\} /\left\{\left[\omega_{322}^{2} \cos \left(\omega_{322} t\right)+\theta_{322}^{2} \cos \left(\theta_{322} t\right)\right]-v_{6}^{2}\left[\cos \left(\omega_{322} t\right)+\right.\right. \\
\left.+\cos \left(\theta_{322} t\right)\right]+\theta_{6}^{2}\left[\omega_{322} \sin \left(\omega_{322} t\right)+\theta_{322} \sin \left(\theta_{322} t\right)\right]
\end{array}\right. \\
& \left\{\begin{array}{c}
z_{312}=\left\{-v_{5}^{2} x_{16} \theta_{16}^{2} h\left[\cos \left(v_{161} t\right)+\cos \left(\theta_{161} t\right)\right]+\theta_{5}^{2} x_{16} \theta_{16}^{2} h\left[v_{161} \sin \left(v_{161} t\right)+\right.\right. \\
\left.\left.\theta_{161} \sin \left(\theta_{161} t\right)\right]\right\} /\left\{\left[\omega_{312}^{2} \cos \left(\omega_{312} t\right)+\theta_{312}^{2} \cos \left(\theta_{312} t\right)\right]-v_{5}^{2}\left[\cos \left(\omega_{312} t\right)+\right.\right. \\
\left.\cos \left(\theta_{312} t\right)\right]+\theta_{5}^{2}\left[\omega_{312} \sin \left(\omega_{312} t\right)+\theta_{312} \sin \left(\theta_{312} t\right)\right] \cdot\left[\cos \left(\omega_{312} t\right)+\cos \left(\theta_{312} t\right)\right] \\
z_{322}=\left\{-v_{6}^{2} x_{16} \theta_{16}^{2} h\left[\cos \left(v_{161} t\right)+\cos \left(\theta_{161} t\right)\right]+\theta_{6}^{2} x_{16} \theta_{16}^{2} h\left[v_{161} \sin \left(v_{161} t\right)+\right.\right. \\
\left.\left.\theta_{161} \sin \left(\theta_{161} t\right)\right]\right\} /\left\{\left[\omega_{322}^{2} \cos \left(\omega_{322} t\right)+\theta_{322}^{2} \cos \left(\theta_{322} t\right)\right]-v_{6}^{2}\left[\cos \left(\omega_{322} t\right)+\right.\right. \\
\left.+\cos \left(\theta_{322} t\right)\right]+\theta_{6}^{2}\left[\omega_{322} \sin \left(\omega_{322} t\right)+\theta_{322} \sin \left(\theta_{322} t\right)\right] \cdot\left[\cos \left(\omega_{322} t\right)+\cos \left(\theta_{322} t\right)\right]
\end{array}\right.
\end{aligned}
$$

As is known, the solution to a homogeneous equation can be represented in the form

$$
\left\{\begin{array}{l}
z_{311}=1 / 2 A_{311} t\left[\sin v_{5} t+\sin \theta_{5} t\right] \\
z_{321}=1 / 2 A_{321} t\left[\sin v_{6} t+\sin \theta_{6} t\right]
\end{array}\right.
$$

Then the general solution of equation (13) is represented as 


$$
\left\{\begin{array}{c}
z_{31}=\left\{-v_{5}^{2} x_{16} \theta_{16}^{2} h\left[\cos \left(v_{161} t\right)+\cos \left(\theta_{161} t\right)\right]+\theta_{5}^{2} x_{16} \theta_{16}^{2} h\left[v_{161} \sin \left(v_{161} t\right)+\right.\right. \\
\left.\left.\theta_{161} \sin \left(\theta_{161} t\right)\right]\right\} /\left\{\left[\omega_{312}^{2} \cos \left(\omega_{312} t\right)+\theta_{312}^{2} \cos \left(\theta_{312} t\right)\right]-v_{5}^{2}\left[\cos \left(\omega_{312} t\right)+\right.\right. \\
\left.\left.\cos \left(\theta_{312} t\right)\right]+\theta_{5}^{2}\left[\omega_{312} \sin \left(\omega_{312} t\right)+\theta_{312} \sin \left(\theta_{312} t\right)\right]\right\} \cdot\left[\cos \left(\omega_{312} t\right)+\cos \left(\theta_{312} t\right)\right]+ \\
1 / 2 A_{311} t\left[\sin v_{5} t+\sin \theta_{5} t\right] \\
z_{32}=\left\{-v_{6}^{2} x_{16} \theta_{16}^{2} h\left[\cos \left(v_{161} t\right)+\cos \left(\theta_{161} t\right)\right]+\theta_{6}^{2} x_{16} \theta_{16}^{2} h\left[v_{161} \sin \left(v_{161} t\right)+\right.\right. \\
\left.\left.\theta_{161} \sin \left(\theta_{161} t\right)\right]\right\} /\left\{\left[\omega_{322}^{2} \cos \left(\omega_{322} t\right)+\theta_{322}^{2} \cos \left(\theta_{322} t\right)\right]-v_{6}^{2}\left[\cos \left(\omega_{322} t\right)+\right.\right. \\
\left.\left.+\cos \left(\theta_{322} t\right)\right]+\theta_{6}^{2}\left[\omega_{322} \sin \left(\omega_{322} t\right)+\theta_{322} \sin \left(\theta_{322} t\right)\right]\right\} \cdot\left[\cos \left(\omega_{322} t\right)+\cos \left(\theta_{322} t\right)\right]+ \\
1 / 2 A_{321} t\left[\operatorname{sinv}_{6} t+\sin \theta_{6} t\right]
\end{array}\right.
$$

The time origin $(\mathrm{t}=\pi / 2)$ in this system can be taken for such a moment when $\mathrm{z}=0$. In this case, substituting in $(\mathrm{t}=\pi / 2$ and $\mathrm{z}=0$, we obtain

$$
\begin{gathered}
\left\{\theta_{5}^{2} x_{16} \theta_{16}^{2} h\left[v_{161} \sin \left(v_{161} \frac{\pi}{2}\right)+\theta_{161} \sin \left(\theta_{161} \frac{\pi}{2}\right)\right]\right\} /\left\{\theta_{5}^{2}\left[\omega_{312} \sin \left(\omega_{312} \frac{\pi}{2}\right)+\theta_{312} \sin \left(\theta_{312} \frac{\pi}{2}\right)\right]\right\}+1 / \\
2 \mathrm{~A}_{311} \frac{\pi}{2}\left[\sin \left(v_{5} \frac{\pi}{2}\right)+\sin \left(\theta_{5} \frac{\pi}{2}\right)\right]=0 \\
\left\{\theta_{6}^{2} x_{16} \theta_{16}^{2} h\left[v_{161} \sin \left(v_{161} \frac{\pi}{2}\right)+\theta_{161} \sin \left(\theta_{161} \frac{\pi}{2}\right)\right]\right\} /\left\{\theta_{6}^{2}\left[\omega_{322} \sin \left(\omega_{322} \frac{\pi}{2}\right)+\theta_{322} \sin \left(\theta_{322} \frac{\pi}{2}\right)\right]\right\}+ \\
1 / 2 \mathrm{~A}_{321} \frac{\pi}{2}\left[\sin v_{6} \frac{\pi}{2}+\sin \theta_{6} \frac{\pi}{2}\right]=0
\end{gathered}
$$

Consequently,

$$
\begin{gathered}
\mathrm{A}_{311}=-\left\{\theta_{5}^{2} x_{16} \theta_{16}^{2} h\left[v_{161} \sin \left(v_{161} \frac{\pi}{2}\right)+\theta_{161} \sin \left(\theta_{161} \frac{\pi}{2}\right)\right]\right\} /\left\{\theta _ { 5 } ^ { 2 } \left[\omega_{312} \sin \left(\omega_{312} \frac{\pi}{2}\right)\right.\right. \\
\left.\left.+\theta_{312} \sin \left(\theta_{312} \frac{\pi}{2}\right)\right]\right\} \cdot \\
\pi\left[\sin \left(v_{5} \frac{\pi}{2}\right)+\sin \left(\theta_{5} \frac{\pi}{2}\right)\right] \\
\mathrm{A}_{321}=-\left\{\theta_{6}^{2} x_{16} \theta_{16}^{2} h\left[v_{161} \sin \left(v_{161} \frac{\pi}{2}\right)+\theta_{161} \sin \left(\theta_{161} \frac{\pi}{2}\right)\right]\right\} \\
/\left\{\theta_{6}^{2}\left[\omega_{322} \sin \left(\omega_{322} \frac{\pi}{2}\right)+\theta_{322} \sin \left(\theta_{322} \frac{\pi}{2}\right)\right]\right\} \cdot \pi\left[\sin v_{6} \frac{\pi}{2}+\sin \theta_{6} \frac{\pi}{2}\right]
\end{gathered}
$$

Substituting A in (15), we obtain

$$
\left\{\begin{array}{c}
z_{31}=\left\{-v_{5}^{2} x_{16} \theta_{16}^{2} h\left[\cos \left(v_{161} t\right)+\cos \left(\theta_{161} t\right)\right]+\theta_{5}^{2} x_{16} \theta_{16}^{2} h\left[v_{161} \sin \left(v_{161} t\right)+\right.\right. \\
\left.\left.\theta_{161} \sin \left(\theta_{161} t\right)\right]\right\} /\left\{\left[\omega_{312}^{2} \cos \left(\omega_{312} t\right)+\theta_{312}^{2} \cos \left(\theta_{312} t\right)\right]-v_{5}^{2}\left[\cos \left(\omega_{312} t\right)+\right.\right. \\
\left.\left.\cos \left(\theta_{312} t\right)\right]+\theta_{5}^{2}\left[\omega_{312} \sin \left(\omega_{312} t\right)+\theta_{312} \sin \left(\theta_{312} t\right)\right]\right\} \cdot\left[\cos \left(\omega_{312} t\right)+\cos \left(\theta_{312} t\right)\right]- \\
1 / 2 t\left[\sin v_{5} t+\sin \theta_{5} t\right] \cdot\left\{\theta_{5}^{2} x_{16} \theta_{16}^{2} h\left[v_{161} \sin \left(v_{161} \frac{\pi}{2}\right)+\theta_{161} \sin \left(\theta_{161} \frac{\pi}{2}\right)\right]\right\} / \\
\left\{\theta_{5}^{2}\left[\omega_{312} \sin \left(\omega_{312} \frac{\pi}{2}\right)+\theta_{312} \sin \left(\theta_{312} \frac{\pi}{2}\right)\right]\right\} \cdot \pi\left[\sin \left(v_{5} \frac{\pi}{2}\right)+\sin \left(\theta_{5} \frac{\pi}{2}\right)\right] \\
z_{32}=\left\{-v_{6}^{2} x_{16} \theta_{16}^{2} h\left[\cos \left(v_{161} t\right)+\cos \left(\theta_{161} t\right)\right]+\theta_{6}^{2} x_{16} \theta_{16}^{2} h\left[v_{161} \sin \left(v_{161} t\right)+\right.\right. \\
\left.\left.\theta_{161} \sin \left(\theta_{161} t\right)\right]\right\} /\left\{\left[\omega_{322}^{2} \cos \left(\omega_{322} t\right)+\theta_{322}^{2} \cos \left(\theta_{322} t\right)\right]-v_{6}^{2}\left[\cos \left(\omega_{322} t\right)+\right.\right. \\
\left.\left.+\cos \left(\theta_{322} t\right)\right]+\theta_{6}^{2}\left[\omega_{322} \sin \left(\omega_{322} t\right)+\theta_{322} \sin \left(\theta_{322} t\right)\right]\right\} \cdot\left[\cos \left(\omega_{322} t\right)+\cos \left(\theta_{322} t\right)\right]- \\
1 / 2 t\left[\sin v_{6} t+\sin \theta_{6} t\right] \cdot\left\{\theta_{6}^{2} x_{16} \theta_{16}^{2} h\left[v_{161} \sin \left(v_{161} \frac{\pi}{2}\right)+\theta_{161} \sin \left(\theta_{161} \frac{\pi}{2}\right)\right]\right\} / \\
\left\{\theta_{6}^{2}\left[\omega_{322} \sin \left(\omega_{322} \frac{\pi}{2}\right)+\theta_{322} \sin \left(\theta_{322} \frac{\pi}{2}\right)\right]\right\} \cdot \pi\left[\sin v_{6} \frac{\pi}{2}+\sin \theta_{6} \frac{\pi}{2}\right]
\end{array}\right.
$$

With the approximation of $\omega$ to $v, \theta$, the period $T_{5}, T_{6}$ increases. When the quantities $\omega$ and $v, \theta$ exactly coincide, the phenomenon of resonance sets in. 
c) equations describing the vertical vibrations of the tractor operator's seat, taking into account the walking of the tractor on an uneven road.

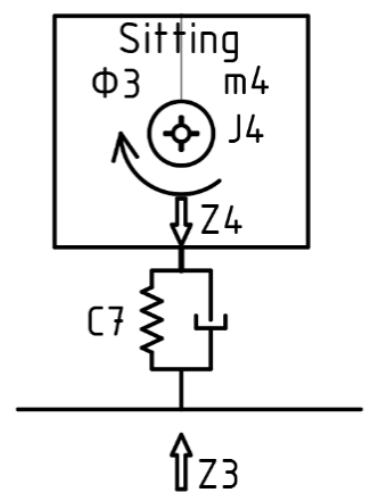

Fig. 4. Vertical oscillation of the operator's seat

for the seat (11)

$$
m_{4} \ddot{z}_{41}+c_{7}\left(z_{4}-z_{3}\right)+k_{7}\left(\dot{z}_{4}-\dot{z}_{3}\right)=0
$$

If from (16) we take $z_{3}=z_{32}$, then

Dividing all the terms of this equation by $\mathrm{m}_{4}$, we get

$$
\ddot{z}_{4}+v_{7}^{2}\left(z_{4}-z_{3}\right)+\theta_{7}^{2}\left(\dot{z}_{4}-\dot{z}_{3}\right)=0
$$

Where $v_{7}^{2}=\frac{c_{7}}{m_{4}}, \theta_{7}^{2}=\frac{k_{7}}{m_{4}}$ is the circular frequency of free vibrations of the system.

The general solution of this equation with the right-hand side (inhomogeneous) can be represented as the sum of the solution to the homogeneous equation $z_{41}$ and the particular solution to the inhomogeneous equation $\boldsymbol{z}_{42}$, i.e., $\boldsymbol{z}_{4}=\boldsymbol{z}_{41}+\boldsymbol{z}_{42}$.

Let us first find a particular solution to the equation. Imagine that

$$
z_{42}=A_{42}\left[\cos \left(\omega_{42} t\right)+\cos \left(\theta_{42} t\right)\right]
$$

and substitute it into equation (18). As a result, we get

$$
\begin{gathered}
-A_{42}\left[\omega_{42}^{2} \cos \left(\omega_{42} t\right)+\theta_{42}^{2} \cos \left(\theta_{42} t\right)\right]+v_{7}^{2} A_{42}\left[\cos \left(\omega_{42} t\right)+\cos \left(\theta_{42} t\right)\right]-v_{7}^{2} z_{32} \\
+k_{7} A_{42}\left[\omega_{42} \sin \left(\omega_{42} t\right)+\theta_{42} \sin \left(\theta_{42} t\right)\right]-k_{7} \dot{z}_{32}=0
\end{gathered}
$$

Where

$$
\begin{aligned}
& A_{42}=\left[k_{7} \dot{z}_{32}+v_{7}^{2} z_{32}\right] /\left\{\left[\omega_{42}^{2} \cos \left(\omega_{42} t\right)+\theta_{42}^{2} \cos \left(\theta_{42} t\right)\right]\right. \\
& \left.\quad+v_{7}^{2}\left[\cos \left(\omega_{42} t\right)+\cos \left(\theta_{42} t\right)\right]+k_{7}\left[\omega_{42} \sin \left(\omega_{42} t\right)+\theta_{42} \sin \left(\theta_{42} t\right)\right]\right\} \\
& z_{42}=\left[k_{7} \dot{z}_{32}+v_{7}^{2} z_{32}\right] /\left\{\left[\omega_{42}^{2} \cos \left(\omega_{42} t\right)+\theta_{42}^{2} \cos \left(\theta_{42} t\right)\right]\right. \\
& \left.\quad+v_{7}^{2}\left[\cos \left(\omega_{42} t\right)+\cos \left(\theta_{42} t\right)\right]+k_{7}\left[\omega_{42} \sin \left(\omega_{42} t\right)+\theta_{42} \sin \left(\theta_{42} t\right)\right]\right\} \\
& \quad \cdot \cos \left(\omega_{42} t\right)
\end{aligned}
$$

As is known, the solution to a homogeneous equation can be represented in the form

$$
z_{41}=\mathrm{A}_{41} t\left[\sin v_{7} t+\sin \theta_{7} t\right]
$$


Then the general solution of equation (18) is represented as

$$
\begin{gathered}
z_{4}= \\
{\left[k_{7} \dot{z}_{32}+v_{7}^{2} z_{32}\right] /\left\{\left[\omega_{42}^{2} \cos \left(\omega_{42} t\right)+\theta_{42}^{2} \cos \left(\theta_{42} t\right)\right]+\right.} \\
\left.v_{7}^{2}\left[\cos \left(\omega_{42} t\right)+\cos \left(\theta_{42} t\right)\right]+k_{7}\left[\omega_{42} \sin \left(\omega_{42} t\right)+\theta_{42} \sin \left(\theta_{42} t\right)\right]\right\} \cdot \cos \left(\omega_{42} t\right)+\mathrm{A}_{41} t\left[\sin v_{7} t+\right. \\
\left.\sin \theta_{7} t\right](21)
\end{gathered}
$$

The time origin $(\mathrm{t}=\pi / 2)$ in this system can be taken for such a moment when $\mathrm{z}=0$. In this case, substituting $\mathrm{t}=\pi / 2$ and $\mathrm{z}=0$ into $(21)$, we obtain

$$
\begin{gathered}
{\left[k_{7} \dot{z}_{32}\right] /\left\{\left[\omega_{42}^{2} \cos \left(\omega_{42} t\right)+\theta_{42}^{2} \cos \left(\theta_{42} t\right)\right]+v_{7}^{2}\left[\cos \left(\omega_{42} t\right)+\cos \left(\theta_{42} t\right)\right]+k_{7}\left[\omega_{42}+\theta_{42}\right]\right\}} \\
\cdot \cos \left(\omega_{42} t\right)+\mathrm{A}_{41} t\left[\sin v_{7} t+\sin \theta_{7} t\right]
\end{gathered}
$$

Consequently,

$$
\begin{gathered}
\mathrm{A}_{41}=-\left[k_{7} \dot{z}_{32}\right] /\left\{\left[\omega_{42}^{2} \cos \left(\omega_{42} t\right)+\theta_{42}^{2} \cos \left(\theta_{42} t\right)\right]+v_{7}^{2}\left[\cos \left(\omega_{42} t\right)+\cos \left(\theta_{42} t\right)\right]+k_{7}\left[\omega_{42}+\theta_{42}\right]\right\} \\
\cdot \cos \left(\omega_{42} t\right) \cdot \quad t\left[\sin v_{7} t+\sin \theta_{7} t\right]
\end{gathered}
$$

Substituting $\mathrm{A}_{41}$ in (21), we obtain

$$
\begin{aligned}
& z_{4}=\left[k_{7} \dot{z}_{32}+v_{7}^{2} z_{32}\right] /\left\{\left[\omega_{42}^{2} \cos \left(\omega_{42} t\right)+\theta_{42}^{2} \cos \left(\theta_{42} t\right)\right]\right. \\
&+\left.v_{7}^{2}\left[\cos \left(\omega_{42} t\right)+\cos \left(\theta_{42} t\right)\right]+k_{7}\left[\omega_{42} \sin \left(\omega_{42} t\right)+\theta_{42} \sin \left(\theta_{42} t\right)\right]\right\} \\
& \cdot \cos \left(\omega_{42} t\right)+ \\
&=-\left[k_{7} \dot{z}_{32}\right] /\left\{\left[\omega_{42}^{2} \cos \left(\omega_{42} t\right)+\theta_{42}^{2} \cos \left(\theta_{42} t\right)\right]\right. \\
&\left.+v_{7}^{2}\left[\cos \left(\omega_{42} t\right)+\cos \left(\theta_{42} t\right)\right]+k_{7}\left[\omega_{42}+\theta_{42}\right]\right\} \cdot \cos \left(\omega_{42} t\right) \\
& \cdot t\left[\sin v_{7} t+\sin \theta_{7} t\right] \cdot t\left[\sin v_{7} t+\sin \theta_{7} t\right]
\end{aligned}
$$

Finally, we define the movements of the tractor seat

$$
\left\{\begin{array}{c}
z_{32}=\left\{-v_{6}^{2} x_{16} \theta_{16}^{2} h\left[\cos \left(v_{161} t\right)+\cos \left(\theta_{161} t\right)\right]+\theta_{6}^{2} x_{16} \theta_{16}^{2} h\left[v_{161} \sin \left(v_{161} t\right)+\right.\right. \\
\left.\left.\theta_{161} \sin \left(\theta_{161} t\right)\right]\right\} /\left\{\left[\omega_{322}^{2} \cos \left(\omega_{322} t\right)+\theta_{322}^{2} \cos \left(\theta_{322} t\right)\right]-v_{6}^{2}\left[\cos \left(\omega_{322} t\right)+\right.\right. \\
\left.\left.+\cos \left(\theta_{322} t\right)\right]+\theta_{6}^{2}\left[\omega_{322} \sin \left(\omega_{322} t\right)+\theta_{322} \sin \left(\theta_{322} t\right)\right]\right\} \cdot\left[\cos \left(\omega_{322} t\right)+\cos \left(\theta_{322} t\right)\right]- \\
1 / 2 t\left[\sin _{6} t+\sin \theta_{6} t\right] \cdot\left\{\theta_{6}^{2} x_{16} \theta_{16}^{2} h\left[v_{161} \sin \left(v_{161} \frac{\pi}{2}\right)+\theta_{161} \sin \left(\theta_{161} \frac{\pi}{2}\right)\right]\right\} / \\
\left\{\theta_{6}^{2}\left[\omega_{322} \sin \left(\omega_{322} \frac{\pi}{2}\right)+\theta_{322} \sin \left(\theta_{322} \frac{\pi}{2}\right)\right]\right\} \cdot \pi\left[\sin v_{6} \frac{\pi}{2}+\sin \theta_{6} \frac{\pi}{2}\right] \\
z_{4}=\left[k_{7} \dot{z}_{32}+v_{7}^{2} z_{32}\right] /\left\{\left[\omega_{42}^{2} \cos \left(\omega_{42} t\right)+\theta_{42}^{2} \cos \left(\theta_{42} t\right)\right]+\right. \\
\left.v_{7}^{2}\left[\cos \left(\omega_{42} t\right)+\cos \left(\theta_{42} t\right)\right]+k_{7}\left[\omega_{42} \sin \left(\omega_{42} t\right)+\theta_{42} \sin \left(\theta_{42} t\right)\right]\right\} \cdot \\
\cos \left(\omega_{42} t\right)+=-\left[k_{7} \dot{z}_{32}\right] /\left\{\left[\omega_{42}^{2} \cos \left(\omega_{42} t\right)+\theta_{42}^{2} \cos \left(\theta_{42} t\right)\right]+\right. \\
\left.+v_{7}^{2}\left[\cos \left(\omega_{42} t\right)+\cos \left(\theta_{42} t\right)\right]+k_{7}\left[\omega_{42}+\theta_{42}\right]\right\} \cdot \cos \left(\omega_{42} t\right) \cdot \\
t\left[\sin v_{7} t+\sin \theta_{7} t\right] \cdot t\left[\sin v_{7} t+\sin \theta_{7} t\right]
\end{array}\right.
$$

The value $\frac{x_{6} v_{6}^{2} v_{7}^{2}}{\left(v_{7}^{2}-\omega_{42}^{2}\right)}=x_{7}$ is called the coefficient of growth of oscillations. Taking this notation, equation (22) will be written

$$
z_{4}=3 / 4 x_{7} h t\left(\sin \varepsilon_{2} t+\operatorname{sinv}_{6} t+\sin _{7} t\right)
$$

As usual, with the approximation of $\omega$ to $v, \theta$, the period $T_{7}$ increases. When the quantities $\omega$ and $v, \theta$ coincide exactly, the phenomenon of resonance occurs. 


\section{Conclusions}

For the first time, the hierarchical link of the tractor was mathematically modeled, taking into account the unevenness of the roads based on the coefficient of stiffness and damping. The equations describe the vertical and longitudinal-angular vibrations of the main parts of the tractor. The hierarchical vertical linear oscillation of the tractor is mathematically modeled, taking into account the unevenness of the roads, the coefficient of stiffness and damping, which serve in the design of wheeled machines, is considered. The use of this mathematical model will make it possible to determine in advance the possible fluctuations of the new designed design of machines. It will help to optimize their design and dynamic parameters.

\section{References}

1. Sazonov I.S., Kim V.A., Amelchenko N.P., Bilyk O.V., Bilyk S.Yu. Damping of lowfrequency vibrations on the driver's seat of a wheeled tractor. Bulletin of the Belarusian-Russian University. 2014. 4 (45) -S.62.

2. Deine, F. Die Wirkung mechanischer Schwingungen in Kraftfahrzeugen auf den Menschen ATZ 59, F. Deine, D. Dieckmann. - Salzburg: Union of Engineers, 302 p. (1957)

3. hernyshev K.V., Ryabov I.M., Pozdeev A.V., Pylinskaya T.V. Analysis of the principles of damping regulation "skyhook" and "groundhook" in the car suspension. Truck 10. - pp. 3-6. (2018).

4. Amelchenko, N.P. Wheel tractor seat suspension, p. 320. Mogilev, (2006).

5. Mitschke, M. Beitragzur Untersuchung der Fahrzeugsch wingungen (Theorie und Versuch), Deutsche Kraftfahrtforschung und StraBen-verkehrstechnik, Heft p 157 (1962).

6. Lurie A.B. Static dynamics of agricultural aggregates, -L .: - 376 p. Kolos, (1970).

7. Control of the movement of wheeled vehicles based on the measurement and analysis of power factors: article / IS Sazonov [et al.]. - Mechanics of machines, mechanisms and materials. 3 (20). - pp. 177-188. (2012).

8. Sazonov I.S. Dynamic regulation of modes of movement of all-wheel drive wheeled vehicles: monograph.-Minsk: BGPA, -p. 185. (2001).

9. Sazonov I.S. Dynamics of wheeled vehicles: monograph, p. 467. Mogilev, (2006) .

10. Deine, F. Die Wirkung mechanischer Schwingungen in Kraftfahrzeugen auf den Menschen ATZ 59. F. Deine, D. Dieckmann. - Salzburg: Union of Engineers, --p. 302. (1957).

11. Amelchenko, N. P. Suspension of the driver's seat of a wheeled tractor, Mogilev: Belorus.-Ros. un-t,.-p. 187. (2007)

12. Sazonov I.S. et al. Dynamics of wheeled vehicles: monograph, Mogilev: Belorus.Ros. un-tp. P 462. , (2006)

13. Kim V.A. On some measures of vibration damping on the seat of a wheeled tractor, Vestn. MSTU.(1). - pp. 77-82. (2001).

14. International scientific and technical conference at the Turin Polytechnic University in Tashkent. (2018).

15. Silaev A.A. Spectral theory of suspension of transport machines, $\mathrm{M}$. Mashinostroenie, - p. 241. (1981).

16. Rothenberg R.V., Vehicle suspension. Oscillation and smoothness. M Mechanical Engineering, -p. 392. (1982).

17. Blinov E.I. Blinov E.I, New concept of the car suspension model, Izvestiya vuzov. Mechanical engineering. -№3. pp.52-62. (2003). 
18. Shekhovtsov K.V. Reducing the level of vibration load in the tractor operator's workplace due to the use of dynamic dampers in the cab suspension system. Author's abstract. dis. Ph.D. Volgograd. -p. 21. (2013).

19. Ermatova D.I, Imomov Sh., Matmurodov F. Mathematical modeling of the interaction of the main parts of a wheel tractor and the numerical determination of the operator's seat oscillation. IOP Conference Series: Earth and Environmental Science. IOP

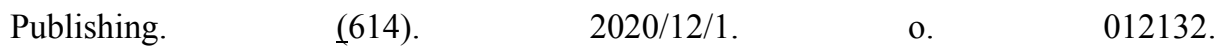
https://iopscience.iop.org/article/10.1088/1755-1315/614/1/012132/meta (2020)

20. Turankhulova D.I, Kamilov A.I, Imamov Sh.D, Matmurodov F.F. Dynamic modeling of vibrating system ns component parts which of that the mobile machines for fast acting pneumatic actuator with self-damping. International Conference on Information Science and Communications Technologies (ICISCT) IEEE. P.1-5. https://ieeexplore.ieee.org/abstract/document/9012010/(2019)

21. Ermatova D.I, Kamilov A.I. Experiment - A field experiment conducted on a designbased damper device for a test tractor. Agroeconomics. pp. 302-306. Tashkent. (2020).

22. Matmurodov F.M. Mathematical spatial modeling of multi-parameter cyclically variable loading of curvilinear turnability and loading of the actuator of a wheeled mobile power plant during a technological operation. Materials of the VII International Scientific and Practical Conference on the topic "Actual problems of innovative development and staffing of the agro-industrial complex". Belarus, Minsk, June 4-5, -pp.220-227. (2020).

23. Sobirov B.Sh., Matmurodov F.M. Mathematical modeling of the dynamic parameters of the action of the power facility and the technological module, J. Bulletin TPUT,. pp.8-11.

24. Stolyarov V.V. Development of an adaptive vibration protection system for a human operator of a shovel excavator of the second size group. Author's abstract. dis. but the competition for the academic degree of candidate of technical sciences. Omsk, -p.18. (2009),

25. Imomov, S., Kholikova, N., Alimova, Z., Nuritov, I., Temirkulova, N. Oil purification devices used in internal combustion engines International Journal of Innovative Technology and Exploring Engineering, 9 (1), pp.3103-3107. (2019).

26. Imomov, Sh.Zh. Heat transfer process during phase back-and-forth motion with biomass pulse loading Applied Solar Energy (English translation of Geliotekhnika), 45 (2), pp. 116-119. (2009).

27. Imomov, Sh.Zh. Engineering design calculation of a biogas unit recuperator Applied Solar Energy (English translation of Geliotekhnika), 43 (3), pp. 196-197. (2007).

28. Imomov, S., Sultonov, M., Aynakulov, S., Usmonov, K., Khafizov, O. Mathematical Model of the Processes of Step-By-Step Processing of Organic Waste International Conference on Information Science and Communications Technologies: Applications, Trends and Opportunities, ICISCT 2019, статья № 9011929, . (2019).

29. Ismatovna, T.D., Ikramovich, K.A., Djaxanovich, I.S., Farhodovich, M.F. Dynamic Modeling of Vibrating System N-S Component Parts Which of That the Mobile Machines for Fast Acting Pneumatic Actuator with Self-Damping (2019) International Conference on Information Science and Communications Technologies: Applications, Trends and Opportunities, ICISCT, статья № 9012010. (2019).

30. Khamidov, F.R., Imomov, S.J., Abdisamatov, O.S., Sarimsaqov, M.M., Ibragimova, G.Kh., Kurbonova, K.I. Optimization of agricultural lands in land equipment projectsJournal of Critical Reviews, 7 (11), pp. 1021-1023. (2020). 
31. Imomov, S., Shodiev, E., Tagaev, V., Qayumov, T, Economic and statistical methods of frequency maintenance of biogas plants IOP Conference Series: Materials Science and Engineering, 883 (1), № 012124, (2020). 